\title{
Finding a sun in building-free graphs
}

\author{
Elaine M. Eschen * Chính T. Hoàng ${ }^{\dagger} \quad$ Jeremy P. Spinrad ${ }^{\ddagger}$ \\ R. Sritharan $\S$
}

November 21, 2018

\begin{abstract}
Deciding whether an arbitrary graph contains a sun was recently shown to be NP-complete [10. We show that whether a building-free graph contains a sun can be decided in $\mathrm{O}\left(\min \left\{m n^{3}, m^{1.5} n^{2}\right\}\right)$ time and, if a sun exists, it can be found in the same time bound. The class of building-free graphs contains many interesting classes of perfect graphs such as Meyniel graphs which, in turn, contains classes such as hhdfree graphs, i-triangulated graphs, and parity graphs. Moreover, there are imperfect graphs that are building-free. The class of buildingfree graphs generalizes several classes of graphs for which an efficient test for the presence of a sun is known. We also present a vertex elimination scheme for the class of (building, gem)-free graphs. The class of (building, gem)-free graphs is a generalization of the class of distance hereditary graphs and a restriction of the class of (building, sun)-free graphs.
\end{abstract}

\section{Introduction}

For a fixed graph $\mathcal{F}$, we say a graph $G$ is $\mathcal{F}$-free if it does not contain $\mathcal{F}$ as an induced subgraph. For a set $\mathcal{S}$ of graphs, we say a graph $G$ is $\mathcal{S}$-free if it

*elaine.eschen@mail.wvu.edu, Lane Department of Computer Science and Electrical Engineering, West Virginia University, Morgantown, WV 26506. Acknowledges support from NSF WV EPSCoR.

${ }^{\dagger}$ choang@wlu.ca, Department of Physics and Computer Science, Wilfrid Laurier University, Waterloo, ON N2L 3C5, Canada. Acknowledges support from NSERC of Canada.

${ }^{\ddagger}$ spin@vuse.vanderbilt.edu, Department of Electrical Engineering and Computer Science, Vanderbilt University, Nashville, TN 37235.

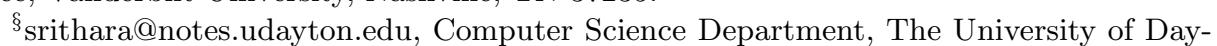
ton, Dayton, OH 45469. Acknowledges support from The National Security Agency, USA. 
does not contain any member of $\mathcal{S}$ as an induced subgraph. When we say "a graph contains $\mathcal{F}$ " ("a graph does not contain $\mathcal{F}$ "), we mean that the graph contains (does not contain) $\mathcal{F}$ as an induced subgraph.

A house is the complement of an induced path on five vertices. A hole is an induced cycle on five or more vertices. A domino is the graph with vertex set $\{a, b, c, d, e, f\}$ and edges $a b, b c, c d, d e, e f, f a$, and be. A graph is chordal if it does not contain any induced cycle on four or more vertices. A sun is a chordal graph with the Hamiltonian cycle $\left(x_{1}, y_{1}, x_{2}, y_{2}, \ldots, x_{n}, y_{n}\right)$, $n \geq 3$, in which each $x_{i}$ is of degree exactly two and the $y_{i}$ vertices form a clique.

The problem of deciding whether a graph contains a sun was recently shown to be NP-complete [10]. However, algorithms exist that can find a sun in polynomial time provided the input graph belongs to a restricted class. A graph is strongly chordal if it is chordal and does not contain a sun [8]. As chordal graphs can be recognized in linear time [16] and as strongly chordal graphs can be recognized in $\mathrm{O}\left(\min \left\{n^{2}, m \log n\right\}\right)$ time [15, 17, it follows that a sun in a chordal graph can be found in $\mathrm{O}\left(\min \left\{n^{2}, m \log n\right\}\right)$ time. A graph is hhd-free if it does not contain a house, hole, or domino as an induced subgraph. The class of hhd-free graphs properly generalizes the class of chordal graphs. The class of hhd-free graphs was originally studied in the context of perfectly orderable graphs [3]. Whether a given graph is hhd-free can be determined in $\mathrm{O}\left(n^{3}\right)$ time [12, 14]. A graph is hhds-free if it is hhd-free and does not contain a sun. It was shown recently that the class of hhds-free graphs can be recognized in $\mathrm{O}\left(n^{3}\right)$ time [7] (the first polynomial-time algorithm for the problem appears in [13]). Thus, deciding whether a given hhd-free graph contains a sun can be done in $\mathrm{O}\left(n^{3}\right)$ time. In light of the hardness of testing for a sun in general [10], determining for which classes of graphs one can test for the presence of suns efficiently is interesting.

In this paper, we show that whether a building-free graph contains a sun can be tested in $\mathrm{O}\left(m n^{3}\right)$ time. A building is the graph obtained from a hole by adding exactly one edge $e$ so that the edge $e$ forms a triangle with two incident edges of the hole. Thus, a house is a building on five vertices. Our algorithm can also find a sun in a given building-free graph, should it exist, in $\mathrm{O}\left(m n^{3}\right)$ time. It is easily seen that every hhds-free graph is building-free. In fact, every Meyniel graph is building-free; a graph is a Meyniel graph if every odd cycle with at least five vertices in the graph has at least two chords. The class of Meyniel graphs is perfect and contains such classes of graphs as hhd-free, i-triangulated, and parity. The class of building-free graphs is rather large and it is not contained in the class of perfect graphs. For 
example, a hole on odd number of vertices is building-free, but is imperfect.

A gem is the graph with vertex set $\{a, b, c, d, e\}$ where $\{a, b, c, d\}$ induces a $P_{4}$ and $e$ is adjacent to each of $a, b, c$, and $d$. We show that every (building, gem)-free graph admits a certain elimination scheme of vertices. The class of (building, gem)-free graphs is a generalization of the class of distance hereditary graphs; distance hereditary graphs are exactly those graphs that are (house, hole, domino, gem)-free. It is seen that every sun contains a gem. Thus, the class of (building, gem)-free graphs is contained in the class of (building, sun)-free graphs.

Our algorithm borrows ideas used in [7] for the recognition of hhds-free graphs. However, in the interest of completeness, we provide full details in our paper, while clearly noting how the ideas from [7] are used. The algorithm in [7, in a sense, reduces the problem of finding a sun in the input graph to finding a sun on six vertices in a derived chordal graph. Deriving such an auxiliary chordal graph and looking for a sun on six vertices in that graph involve properties of strongly chordal graphs and the process of "strongly chordal completion", which is discussed later. In view of this, we next present some known properties of strongly chordal graphs. First, we need some definitions.

We use $N(x)$ to denote the set of vertices adjacent to vertex $x$ in a graph $G$; i.e., $N(x)$ is the set of neighbours of $x$. The set $N(x)$ is referred to as the (open) neighbourhood of $x$, while the set $N[x]=N(x) \cup\{x\}$ is the closed neighbourhood of $x$. The set of nonneighbours of $x$ is denoted by $M(x)=V(G)-N[x]$. For vertices $x$ and $y$ of graph $G$, we say $x$ sees $y$ when $x$ and $y$ are adjacent in $G$, and $x$ misses $y$ when $x$ and $y$ are not adjacent in $G$.

Vertex $x$ in a graph is simplicial if $N(x)$ induces a complete graph. It is well known [4] that graph $G$ is chordal if and only if every induced subgraph $H$ of $G$ contains a simplicial vertex of $H$. Farber [8] proved an analogous characterization for the class of strongly chordal graphs. Vertex $x$ in a graph is simple if the vertices in $N(x)$ can be ordered as $x_{1}, x_{2}, \ldots, x_{k}$ such that $N\left[x_{1}\right] \subseteq N\left[x_{2}\right] \subseteq \ldots \subseteq N\left[x_{k}\right]$. Thus, every simple vertex is simplicial. For a graph $G$, let $\mathcal{R}=v_{1}, v_{2}, \ldots, v_{n}$ be an ordering of vertices of $G$. Let $G(i)=G\left[\left\{v_{i}, v_{i+1}, \ldots, v_{n}\right\}\right]$; i.e., $G(i)$ is the subgraph induced in $G$ by the set of vertices $\left\{v_{i}, v_{i+1}, \ldots, v_{n}\right\}$. $\mathcal{R}$ is a simple elimination ordering for $G$ if $v_{i}$ is simple in $G(i), 1 \leq i \leq n$. $\mathcal{R}$ is a strong elimination ordering for $G$ if (i) $\mathcal{R}$ is a simple elimination ordering, and (ii) for every $i<j<k$ such that $v_{j}, v_{k} \in N\left(v_{i}\right), N\left[v_{j}\right] \subseteq N\left[v_{k}\right]$ in $G(i)$. In other words, in a strong elimination ordering, for any vertex $v_{i}$, the neighbours of $v_{i}$ in $G(i)$ appear in $\mathcal{R}$ according to the order of inclusion of their closed neighbourhoods in 
$G(i)$. The following is due to Farber [8]:

Theorem 1 ([8]) The following are equivalent for any graph $G$ :

- $G$ is strongly chordal.

- $G$ is chordal and does not contain a sun.

- Vertices of $G$ admit a simple elimination ordering.

- Vertices of $G$ admit a strong elimination ordering.

\subsection{The algorithm}

Note that whether an arbitrary graph contains a building can be decided in $\mathrm{O}\left(m n^{3}\right)$ time. For every vertex $v$ of the graph, for every pair $x, y$ of adjacent vertices in $N(v)$, we do the following: delete all the other neighbours of $v$, delete the vertex $v$, delete every vertex in $N(x) \cap N(y)$, delete the edge $x y$, and check if a path connecting $x$ and $y$ exists in the remaining graph. Thus, though we present our algorithm in such a way that it takes a building-free graph as input and tests for the presence of a sun, it can easily be modified so that it takes an arbitrary graph as input and tests for the presence of a sun or a building in the graph. We need some definitions first.

Definition 1 By a k-sun $\left(\left(d_{1}, d_{2}, \ldots, d_{k}\right),\left(c_{1}, c_{2}, \ldots, c_{k}\right)\right)$ with $k \geq 3$ we denote the graph obtained by taking a clique on vertices $c_{1}, c_{2}, \ldots, c_{k}$, a stable set on vertices $d_{1}, d_{2}, \ldots, d_{k}$, and for each $i, 1 \leq i \leq k$, adding edges $d_{i} c_{i}, d_{i} c_{i+1}$ with the subscripts taken modulo $k$. The vertices $d_{i}$ are called the tips of the sun. For an arbitrary $k$, we refer to a $k$-sun simply as a sun.

Definition 2 [7] By a sunflower $\left(\left(P_{1}, P_{2}, \ldots, P_{k}\right),\left(c_{1}, c_{2}, \ldots, c_{k}\right)\right)$ with $k \geq$ 3 we denote the graph obtained by taking an arbitrary graph with vertices $c_{1}, c_{2}, \ldots, c_{k}$, and for each $i, 1 \leq i \leq k$, adding an induced path $P_{i}$ of length at least two connecting $c_{i}$ to $c_{i+1}$ with the subscripts taken modulo $k$. The paths $P_{i}$ are called the petals of the sunflower. The edges of the petals are called boundary edges of the sunflower. Vertices $c_{i}$ are called center vertices, the remaining vertices of the sunflower are called petal vertices. If a path $P_{i}$ has length two, the petal vertex of $P_{i}$ is called a tip of the sunflower.

First we explain the basic logic behind the algorithm. Then, we present the algorithm and its proof of correctness. Suppose $G^{\prime}$ is a building-free graph. If a sun exists in $G^{\prime}$, then some vertex $x$ must be a tip of a sun 
in $G^{\prime}$, and since a sun is a sunflower, $x$ is also a tip of a sunflower in $G^{\prime}$. Further, the only two neighbours of $x$ in this sunflower are adjacent. We will show later that if a vertex $x$ is a tip of a sunflower $S$ in $G^{\prime}$ such that the only two neighbours of $x$ in $S$ are adjacent, then a sun must exist in $G^{\prime}$. Therefore, our algorithm checks every vertex $x$ of $G^{\prime}$ to determine whether $x$ is such a tip of a sunflower in $G^{\prime}$. In order to do this, for every edge $y z$ in the neighbourhood of $x$, we consider the graph $G$ obtained from $G^{\prime}$ by deleting all the neighbours of $x$ except $y$ and $z$, and determine whether $x$ is the tip of a sunflower in $G$. Note that $G$ is building-free, vertex $x$ has only two neighbours in $G$, and $x$ is simplicial in $G$.

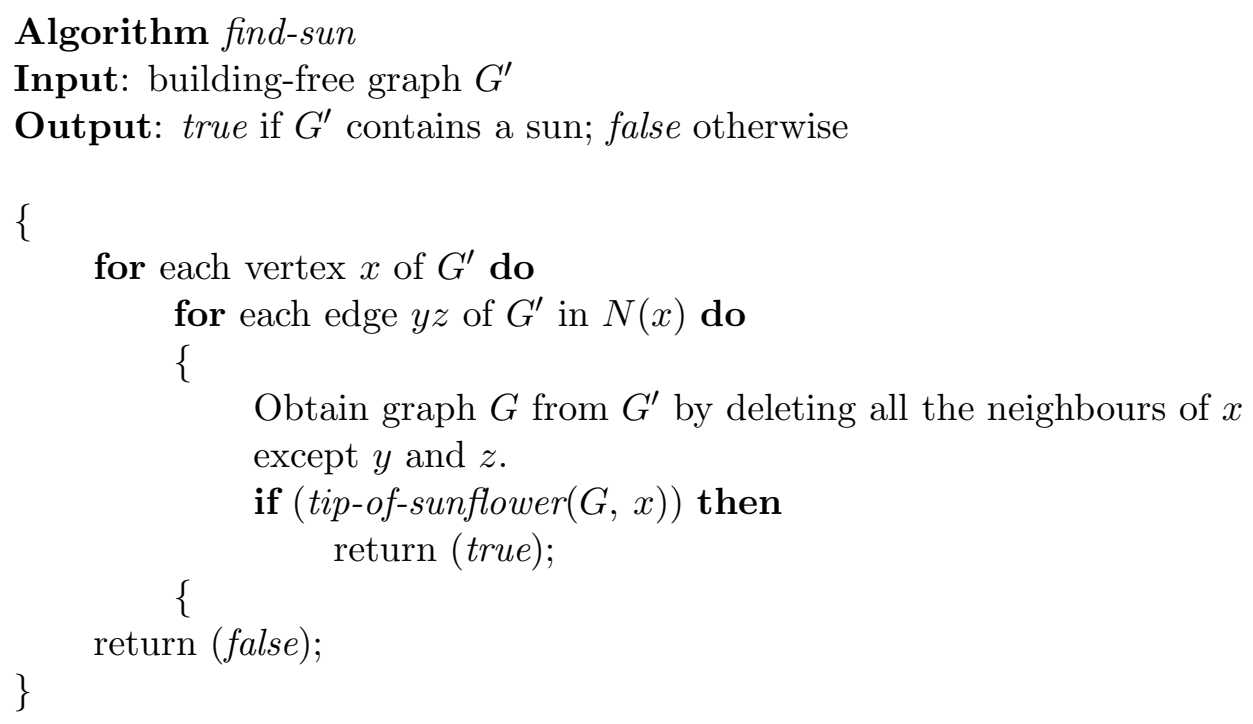

Next, we present algorithm tip-of-sunflower, which was used in the context of hhd-free graphs in [7. We will show that it can be adopted for use in the context of building-free graphs also.

Algorithm tip-of-sunflower

Input: building-free graph $G$ and a simplicial vertex $x$ of $G$

Output: true if $x$ is the tip of a sunflower in $G$; false otherwise

\{

1. For each vertex $y \in M(x)$ compute $n(y, x)=|N(y) \cap N(x)|$.

2. $\quad$ Sort $M(x)$ into $\mathcal{R}=y_{1}, y_{2}, \ldots, y_{k}$ such that $i<j$ implies $n\left(y_{i}, x\right) \leq n\left(y_{j}, x\right)$. 
3. $\quad$ * Perform strongly chordal completion of $G[M(x)]$ */

$S C C(G[M(x)])$;

Obtain graph $H$ from $G$ by adding to $G$ the edges that were

added to $G[M(x)]$ during the strongly chordal completion of $G[M(x)]$. ${ }^{*}$ Now $\mathcal{R}$ is a strong elimination ordering of $H[M(x)] . * /$

4. if $($ in-3-sun $(H, x))$ then

return (true)

else

return (false);

\}

Now we prove some facts about the algorithm tip-of-sunflower. For the sake of proving the facts about the algorithm, we will assume that strongly chordal completion of $G[M(x)]$ in step 3 of Algorithm tip-of-sunflower is performed as in the following $k$ iterations (as given in [7]):

\section{Algorithm $S C C$}

Input: Graph $G=(V, E)$ and an arbitrary ordering $\mathcal{R}$ of $V$

Output: Graph $G_{k}=\left(V, E_{k}\right)$ where $E \subseteq E_{k}$ and

$\mathcal{R}$ is a strong elimination ordering for $G_{k}$.

\{

1. $G_{0}=G$

2. for $i=1$ to $k$ do

$/ /$ We make $y_{i}$ simple in $G_{i}\left[\left\{y_{i}, y_{i+1}, \ldots, y_{k}\right\}\right]$ such that for $i<p<q$

// the following holds with respect to $G_{i}\left[\left\{y_{i}, y_{i+1}, \ldots, y_{k}\right\}\right]$ :

$/ / \quad$ if $y_{p} \in N\left(y_{i}\right)$ and $y_{q} \in N\left(y_{i}\right)$, then $N\left[y_{p}\right] \subseteq N\left[y_{q}\right]$.

Obtain $G_{i}$ via updating $G_{i-1}$ by adding edges as follows:

Let $A=N_{G_{i-1}}\left(y_{i}\right) \cap\left\{y_{i+1}, \ldots, y_{k}\right\}$.

2.1 Form $G_{i}^{1}$ by adding to $G_{i-1}$ edges between the vertices of $A$ so that $A$ is a clique.

2.2 Let the vertices of $A$ be $y_{i_{1}}, y_{i_{2}}, \ldots, y_{i_{t}}$, where $i_{1}<\ldots<i_{t}$.

Let $G_{i}^{2}=G_{i}^{1}$.

for $j=1$ to $t-1$ do

For each pair of vertices $y_{r}, y_{i_{s}}$,

where $y_{r} \in\left\{y_{i+1}, \ldots, y_{k}\right\}$ and $y_{i_{s}} \in A, j+1 \leq s \leq t$,

such that in $G_{i-1}, y_{i_{j}}$ sees $y_{r}$ and $y_{i_{s}}$ misses $y_{r}$,

add edge $y_{r} y_{i_{s}}$ to $G_{i}^{2}$.

$2.3 G_{i}=G_{i}^{2}$ 
Strongly chordal completion, as described in $S C C$, requires $\mathrm{O}\left(n^{3}\right)$ time. It was shown in [7] that it can be implemented to run in $\mathrm{O}\left(n^{2}\right)$ time.

Definition 3 [7] Given a graph $G=(V, E)$, and a set $X \subseteq V$, we say that vertex $u X$-dominates vertex $v$ if $N(v) \cap X \subseteq N[u] \cap X$. We say $u$ dominates $v$ to mean $u$-dominates $v$.

Let $M(x)=\left\{y_{1}, y_{2}, \ldots, y_{k}\right\}$ and $\mathcal{R}$ be the ordering of the vertices of $M(x)$ produced by step 2 of Algorithm tip-of-sunflower. For convenience, we write $y_{i}<y_{j}$ when $i<j$. Note that whether or not a vertex $N(x)$ dominates another vertex is unaffected by the additions of edges as a result of the strongly chordal completion of $G[M(x)]$, as these edges are always between vertices of $M(x)$.

Lemma 1 was proved in [7] for (house, hole)-free graphs. We show it holds for the more general class of building-free graphs as well.

Lemma 1 Let $G$ be a building-free graph with simplicial vertex $x$. Let $y^{\prime}, y^{\prime \prime} \in M(x)$ be such that $y^{\prime}$ comes before $y^{\prime \prime}$ in $\mathcal{R}$. If $G$ has an induced path $P$ connecting $y^{\prime}$ and $y^{\prime \prime}$ all of whose vertices are in $M(x)$ and such that each internal vertex of $P$ is $N(x)$-dominated by $y^{\prime}$ or $y^{\prime \prime}$, then $y^{\prime \prime} N(x)$-dominates $y^{\prime}$ (and hence, $y^{\prime \prime} N(x)$-dominates each vertex of $P$ ).

Proof of Lemma 1. Suppose $G$ has an induced path $P$ that satisfies the hypothesis, but $y^{\prime \prime}$ does not $N(x)$-dominate $y^{\prime}$. Then $y^{\prime}$ sees $a \in N(x)$ that $y^{\prime \prime}$ misses; also, since $n\left(y^{\prime}, x\right) \leq n\left(y^{\prime \prime}, x\right), y^{\prime \prime}$ sees $b \in N(x)$ that $y^{\prime}$ misses. No internal vertex of $P$ sees both $a$ and $b$, since each such vertex is $N(x)$ dominated by $y^{\prime}$ or $y^{\prime \prime}$. In a traversal of $P$ starting at $y^{\prime}$, let $y_{b}$ be the first vertex of $P$ that sees $b$. Vertex $y_{b}$ exists since $y^{\prime \prime}$ sees $b$. In a traversal of $P$ from $y_{b}$ towards $y^{\prime}$, let $y_{a}$ be the first vertex that sees $a$. Vertex $y_{a}$ exists since $y^{\prime}$ sees $a$. Now either there are no vertices between $y_{a}$ and $y_{b}$ on $P$ and $\left\{x, a, b, y_{a}, y_{b}\right\}$ induces a house in $G$, or $\left\{x, a, b, y_{a}, y_{b}\right\}$ along with the vertices on $P$ between $y_{a}$ and $y_{b}$ (all of which must miss both $a$ and $b$ ) induces a building in $G$. In either case, we have a contradiction. Thus, $y^{\prime \prime}$ $N(x)$-dominates $y^{\prime}$ and, by transitivity, $y^{\prime \prime} N(x)$-dominates every vertex on $P$.

Lemma 2 through Lemma 4 were proved in [7] in the context of (house, hole)-free graphs. Given Lemma 1 (for building-free graphs), the proofs of these lemmata given in [7] are valid without modification for building-free graphs. Rather than reproduce the proofs here, we refer the reader to [7]. 
Lemma 2 Let $G$ be a building-free graph with simplicial vertex $x$. Let $H$ be the graph as constructed in Algorithm tip-of-sunflower. Let $x_{1}, \ldots, x_{r}$ be an arbitrary ordering of vertices in $N(x)$. Then, $H$ is chordal with $y_{1}, \ldots, y_{k}, x_{1}, \ldots, x_{r}, x$ as its perfect elimination scheme.

Lemma 3 Let $G$ be a building-free graph with simplicial vertex $x$. Let $H$ be the graph as constructed in Algorithm tip-of-sunflower. If vertex $x$ is in a sun in $G$, then $x$ is in a 3-sun in $H$.

Lemma 4 Let $G$ be a building-free graph with simplicial vertex $x$. Let $H$ be the graph as constructed in Algorithm tip-of-sunflower. If vertex $x$ is in a 3-sun in $H$, then $x$ is in a sunflower in $G$.

Lemma 5 Let $G$ be a building-free graph. Suppose $G$ contains a sunflower $S$ such that some petal $P$ of $S$ has exactly two edges and the only petal vertex of $P$ is simplicial in $S$. Then, $G$ contains a sun.

Proof of Lemma 5. Consider all the sunflowers of $G$ satisfying the conditions of the lemma that have the fewest number of vertices. From these, pick the sunflower $S=\left(\left(P_{1}, P_{2}, \ldots, P_{k}\right),\left(c_{1}, c_{2}, \ldots, c_{k}\right)\right)$ so that $k$ is as small as possible. We may assume that $P_{k}=c_{k} x c_{1}$ and $c_{1}$ sees $c_{k}$. Also, recall that the two endpoints of $P_{i}, 1 \leq i \leq k-1$, are $c_{i}$ and $c_{i+1}$.

Suppose $k=3$. Then, it is seen that $S$ is indeed a 3 -sun (or else there is a building in $G$ ). Therefore, we can assume that $k \geq 4$.

First, we show that every center vertex must see $c_{k}$. It will then follow from symmetry that every center vertex must see $c_{1}$ also.

Consider a $c_{i}$ such that $i \notin\{1, k\}$. Suppose $c_{i}$ misses $c_{k}(i=2, i=k-1$ are possible). If $c_{i}$ misses every center vertex of $S$, then $\left(\left(P_{1}, \ldots, P_{i-1} P_{i}, \ldots, P_{k}\right)\right.$, $\left.\left(c_{1}, \ldots, c_{i-1}, c_{i+1}, \ldots, c_{k}\right)\right)$ is a sunflower on the same set of vertices as $S$ that contains fewer center vertices and in which $x$ is a tip, a contradiction. Therefore, we can assume that $c_{i}$ misses $c_{k}$, but sees some center vertex of $S$.

Suppose $c_{i}$ misses every $c_{j}, i<j<k$. Traversing in the clockwise direction from $c_{1}$, let $c_{t}$ be the first center vertex that $c_{i}$ sees. Suppose $t=1$. If $i=k-1$, then $\left\{x, c_{1}\right\} \cup P_{k-1}$ induces a building. Therefore, $i \neq k-1$. Then, $\left(\left(c_{1} P_{i}, P_{i+1}, \ldots, P_{k}\right),\left(c_{1}, c_{i+1}, \ldots, c_{k}\right)\right)$ is a smaller sunflower in which $x$ is a tip. Now suppose $1<t<i$. Then, $\left(\left(P_{1}, \ldots, P_{t-1}, c_{t} P_{i}, P_{i+1}, \ldots, P_{k}\right),\left(c_{1}, \ldots\right.\right.$, $\left.\left.c_{t-1}, c_{t}, c_{i+1}, \ldots, c_{k}\right)\right)$ is a smaller sunflower in which $x$ is a tip.

Now we can assume that $c_{i}$ sees some $c_{j}$ such that $i<j<k$ (and therefore, $i \neq k-1)$. Let $c_{t}$ be the first center vertex in the counter clockwise direction from $c_{k}$ that $c_{i}$ sees such that $i<t<k$. If $c_{i}$ has no neighbour $c_{j}$ such 
that $1 \leq j<i$, then $\left(\left(P_{1}, \ldots, P_{i-1} c_{t}, P_{t}, \ldots, P_{k}\right),\left(c_{1}, \ldots, c_{i-1}, c_{t}, \ldots, c_{k}\right)\right)$ is a smaller sunflower in which $x$ is a tip. Otherwise, let $c_{r}$ be the first center vertex in the clockwise direction from $c_{1}$ that $c_{i}$ sees such that $1 \leq r<i$. If $r=1$, then $\left(\left(c_{1} c_{i} c_{t}, P_{t}, \ldots, P_{k}\right),\left(c_{1}, c_{t}, \ldots, c_{k}\right)\right)$ is a smaller sunflower in which $x$ is a tip. On the other hand, if $r \neq 1$, then $\left(\left(P_{1}, \ldots, P_{r-1}, c_{r} c_{i} c_{t}, P_{t}, \ldots, P_{k}\right)\right.$, $\left.\left(c_{1}, \ldots, c_{r-1}, c_{r}, c_{t}, \ldots, c_{k}\right)\right)$ is a smaller sunflower in which $x$ is a tip.

We can now conclude that every center vertex sees $c_{k}$ and every center vertex sees $c_{1}$ also.

Next, we want to show that for $1 \leq i \leq k-1, c_{i}$ sees $c_{i+1}$ and every $P_{i}$ must have exactly two edges. Whenever such a $P_{i}$ is established to have exactly two edges, $d_{i}$ will refer to the only petal vertex of $P_{i}$.

We already have established that $c_{1}$ sees $c_{2}$ and $c_{k-1}$ sees $c_{k}$. Note that for any $i$, if $c_{i}$ sees $c_{i+1}$ then $P_{i}$ has length two; for otherwise, $P_{i} \cup\left\{c_{k}\right\}$ or $P_{i} \cup\left\{c_{1}\right\}$ induces a building. Let $i$ be the smallest subscript (if it exists) such that $c_{i}$ misses $c_{i+1}$. Now, if $c_{i-1}$ sees $c_{i+1}$, then $P_{i} \cup\left\{c_{i-1}, d_{i-1}\right\}$ induces a building; otherwise, $P_{i} \cup\left\{c_{k}, c_{i-1}\right\}$ induces a building.

Finally, it can now be seen that any $d_{i}$ can play the role of vertex $x$. Therefore, applying the same arguments to every $d_{i}$ we can conclude that the set of center vertices forms a clique and $S$ is indeed a sun.

We note that an efficient algorithm to find a sun in a building-free graph, when it exists, can be extracted from the proofs of Lemma 4 and Lemma 5 ,

We now consider Algorithm in-3-sun, which is called by Algorithm tipof-sunflower. The algorithm in-3-sun is reproduced below from [7] for the sake of completeness. However, we refer the reader to [7] for the details on its correctness and complexity.

Algorithm in-3-sun

Input: Chordal graph $H$, simplicial vertex $x$ of $H$, and ordering $\mathcal{R}=y_{1}, y_{2}, \ldots, y_{k}$ of $M(x)$ such that $\mathcal{R}$ is a strong elimination ordering for $H[M(x)]$, for $i<j,\left|N\left(y_{i}\right) \cap N(x)\right| \leq\left|N\left(y_{j}\right) \cap N(x)\right|$, and for $i<j$ with $y_{i} y_{j} \in E(H),\left(N\left(y_{i}\right) \cap N(x)\right) \subseteq\left(N\left(y_{j}\right) \cap N(x)\right)$.

Output: true if $x$ is in a 3 -sun in $H$; false otherwise

\{

1. for $i=1$ to $k-2$ do

For $i<p<q$ such that $y_{i} y_{q} y_{p}$ is a $P_{3}$ in $H$, mark $\left[y_{i}, y_{p}\right]$ as a red edge to be added.

end

2. Add all the red edges to $H$ to obtain $H^{\prime}$. 
3. Let $x_{1}, x_{2}, \ldots, x_{r}$ be an arbitrary ordering of $N(x)$.

if $y_{1}, \ldots, y_{k}, x_{1}, \ldots, x_{r}, x$ is a perfect elimination scheme for $H^{\prime}$ then return (false)

else

return (true);

\}

Lemma 6 [7] Let $G$ be a strongly chordal graph with the strong elimination ordering $\mathcal{S}=v_{1}, v_{2}, \ldots, v_{n}$. Let $G^{\prime}$ be the graph obtained from $G$ by adding every edge $v_{i} v_{j}$ such that, for some $k$ with $i<j<k, v_{i} v_{k} v_{j}$ is a $P_{3}$ in $G$. Then, $G^{\prime}$ is a chordal graph and $\mathcal{S}$ is a perfect elimination scheme for $G^{\prime}$.

Lemma 7 [7] Vertex $x$ is in a 3-sun in $H$ if and only if for some red edge $y_{i} y_{j}$ added with $i<j, y_{j}$ does not $N(x)$-dominate $y_{i}$.

Lemma 8 [7] Vertex $x$ is in a 3 -sun in $H$ if and only if $\mathcal{S}=y_{1}, \ldots, y_{k}, x_{1}, \ldots, x_{r}, x$ is not a perfect elimination scheme for $H^{\prime}$.

Corollary 1 [7] $\mathcal{S}=y_{1}, \ldots, y_{k}, x_{1}, \ldots x_{r}, x$ is not a perfect elimination scheme for $H^{\prime}$ if and only if there exists $y_{i} \in M(x)$ such that $y_{j} \in M(x)$ is the first neighbour of $y_{i}$ in $H^{\prime}$ that comes after it in $\mathcal{R}$ and there exists $w \in N(x)$ that $y_{i}$ sees but $y_{j}$ misses.

\subsection{Time complexity}

Lemma 9 [7] Let $G$ be a graph and $\mathcal{R}=v_{1}, v_{2}, \ldots, v_{n}$ be an arbitrary ordering of its vertices. Then, strongly chordal completion of $G$ ensuring that $\mathcal{R}$ is a strong elimination ordering for the resulting graph can be performed in $\mathrm{O}\left(n^{2}\right)$ time.

Lemma 10 [7] Algorithm in-3-sun can be implemented to run in $\mathrm{O}\left(n^{2}\right)$ time.

Lemma 11 [7] Algorithm tip-of-sunflower can be implemented to run in $\mathrm{O}\left(n^{2}\right)$ time. 
Proof of Lemma 11. Steps 1 and 2 can easily be done in linear time. By Lemma 9, step 3 can be done in $\mathrm{O}\left(n^{2}\right)$ time. Finally, by Lemma 10, step 4 can also be done in $\mathrm{O}\left(n^{2}\right)$ time.

Theorem 2 Algorithm find-sun is correct and it runs in $\mathrm{O}\left(\min \left\{m n^{3}, m^{1.5} n^{2}\right\}\right)$ time.

Proof of Theorem 2. It is clear from Lemma 3, Lemma 4, and Lemma 5 that $G^{\prime}$ contains a sun if and only if for some vertex $x$ and some pair of adjacent neighbours $y$ and $z$ of $x, x$ is a tip of a sunflower in the graph $G$ constructed by the algorithm. Therefore, the algorithm is correct.

The algorithm find-sun invokes algorithm tip-of-sunflower $\mathrm{O}(\mathrm{mn})$ times. Since each such invocation costs $\mathrm{O}\left(n^{2}\right)$ time, the overall time complexity of the algorithm is $\mathrm{O}\left(m n^{3}\right)$.

Since all the triangles of a graph can be listed in $\mathrm{O}\left(\mathrm{m}^{1.5}\right)$ time [1, finding a building as well as algorithm find-sun can be implemented to run in $\mathrm{O}\left(m^{1.5} n^{2}\right)$ time also.

\section{A class of (building, sun)-free graphs}

Several classes of graphs have been characterized via the presence of an elimination scheme of vertices [2]. There are also graph classes that are known to admit an interesting elimination scheme of vertices, though the presence of such a scheme may not characterize the class of graphs.

A bull is the graph constructed by starting with a $P_{4}$ abcd and then adding vertex $e$ that is adjacent only to the vertices $b$ and $c$. The vertex $e$ is said to be the nose of the bull.

In this section we consider the class of (building, gem)-free graphs. It is seen that every distance hereditary graph ((house, hole, domino, gem)free graph) is (building, gem)-free and that every (building, gem)-free graph is (building, sun)-free. We show that every (building, gem)-free graph admits an elimination scheme of vertices that generalizes a known elimination scheme of vertices [6] for distance hereditary graphs. We show that, as in the case of distance hereditary graphs, a lexicographic breadth first search (LBFS) can be used to generate the elimination scheme. Next, we reproduce the details of LBFS from [9]. It is well known that LBFS on any graph can be implemented to run in linear time [16].

Algorithm $L B F S$

Input: graph $G$ 
Output: ordering $\sigma=v_{1} v_{2} \ldots v_{n}$ of vertices of $G$

\{

Assign the label $\emptyset$ to each vertex.

for $i=n$ downto 1 do

\{

Choose an unnumbered vertex $w$ with the largest label.

$v_{i}=w$

for each unnumbered neighbour $x$ of $w$ do

\}

Append $i$ to the label of $x$.

\}

We refer to the output $\sigma$ of LBFS on graph $G$ as an LBFS ordering of $G$. For such an ordering $\sigma$, we say " $v_{i}<v_{j}$ " and " $v_{j}$ is to the right of $v_{i}$ " to mean vertex $v_{i}$ precedes vertex $v_{j}$ in $\sigma$. The following well-known property [16] of an LBFS ordering of a graph $G=(V, E)$ will be used repeatedly:

$\left(P_{*}\right)$ For vertices $a, b, c \in V$, if $a<b<c, a c \in E$, and $b c \notin E$, then there exists $d \in V$ such that $c<d, b d \in E$, and $a d \notin E$.

We refer to an ordered triple $(a, b, c)$ of vertices that satisfies the conditions of $\left(P_{*}\right)$ as an extendable triple.

Definition 4 An extended bull is the graph obtained starting from an induced path $a_{i} \ldots a_{1} b_{1} \ldots b_{j}$, where $i \geq 2$ and $j \geq 2$, and adding vertex $x$ that sees only $a_{1}$ and $b_{1}$. Therefore, when $i=2$ and $j=2$, an extended bull is a bull. Vertex $x$ is the nose of the extended bull. We refer to such an extended bull (bull) as $B\left(x, a_{i}, b_{j}\right)\left(B\left(x, a_{2}, b_{2}\right)\right)$.

Definition 5 Suppose $\sigma$ is an ordering of vertices of graph $G$ produced by LBFS. For a vertex $x$, let $H_{x}$ be the subgraph of $G$ induced by $x$ and the vertices to the right of $x$ in $\sigma$. By " $B\left(x, a_{2}, b_{2}\right)$ is the rightmost bull in which $x$ is the nose" we mean $B\left(x, a_{2}, b_{2}\right)$ is a bull in $H_{x}$ and no vertex to the right of a member of $\left\{a_{2}, a_{1}, b_{1}, b_{2}\right\}$ in $\sigma$ and $x$ are in a bull in $H_{x}$ in which $x$ is the nose.

Definition 6 A near building is a graph $G=\left(\left\{v, w_{1}, \ldots, w_{j}\right\}(j \geq 4), E\right)$ such that $\left\{v w_{1}, v w_{j}, w_{1} w_{j}\right\} \subset E, w_{i} w_{i+1} \in E$ for all $1 \leq i \leq j-1$, and the only other edges in $E$, if they exist, are of the form $w_{j} w_{i}$ where $2 \leq i \leq j-2$. 


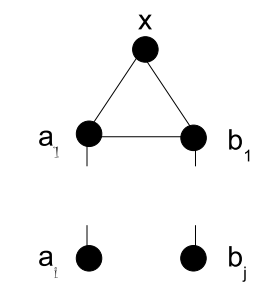

Figure 1: An extended bull in which $x$ is the nose. We have $i \geq 2, j \geq 2$, and each of $a_{1} \ldots a_{i}$ and $b_{1} \ldots b_{j}$ is an induced path.

Claim 1 Let $G$ be a (building, gem)-free graph. Then $G$ cannot contain a near building as an induced subgraph.

Proof of Claim 1. Suppose $G$ contains an induced near building. If $w_{j}$ misses $w_{2}$, then $\left\{v, w_{j}, w_{1}, \ldots, w_{i}\right\}$, where $w_{i}$ is the smallest indexed vertex that $w_{j}$ sees after $w_{1}$, induces a building in $G$. If $w_{j}$ sees $w_{2}$ and $w_{3}$, then $G$ contains an induced gem. If $w_{j}$ sees $w_{2}$ and misses $w_{3}$, then $\left\{w_{1}, w_{j}, w_{2}, \ldots, w_{i}\right\}$, where $w_{i}$ is the smallest indexed vertex that $w_{j}$ sees after $w_{2}$, induces a building in $G$. In each case a contradiction is reached; therefore, $G$ cannot contain an induced near building.

We need the following lemma.

Lemma 12 Suppose $G$ is a (building, gem)-free graph and $\sigma$ is an ordering of vertices of $G$ produced by LBFS. Suppose vertex $x$ is the nose of a bull in $H_{x}$. Further, suppose the following hold:

- $B\left(x, a_{2}, b_{2}\right)$ is the rightmost bull in which $x$ is the nose.

- Vertex $x$ is the nose of the extended bull $B\left(x, a_{i}, b_{j}\right)$ in $H_{x}$ where $i \geq 2$ and $j \geq 2$.

- For all $r, 2 \leq r \leq i-1, a_{r}<a_{r+1}$ in $\sigma$.

- For all $r, 2 \leq r \leq j-1, b_{r}<b_{r+1}$ in $\sigma$. 
- When $i+1=3$, at least one of the following holds: $a_{i+1}$ misses $x$, $a_{i+1}$ misses $b_{1}$, or $a_{1}<a_{i+1}$.

- When $j+1=3$, at least one of the following holds: $b_{j+1}$ misses $x$, $b_{j+1}$ misses $a_{1}$, or $b_{1}<b_{j+1}$.

- For every $k, 4 \leq k \leq i, a_{k}$ is the rightmost vertex in $\sigma$ that sees $a_{k-1}$ and misses every $b_{r}, 1 \leq r \leq j$.

- For every $k, 4 \leq k \leq j, b_{k}$ is the rightmost vertex in $\sigma$ that sees $b_{k-1}$ and misses every $a_{r}, 1 \leq r \leq i$.

- Either $a_{3}$ is the rightmost vertex in $\sigma$ that sees $a_{2}$ and misses $x$ or $a_{3}$ is the rightmost vertex in $\sigma$ that sees $a_{2}$ and misses every $b_{r}, 1 \leq r \leq j$.

- Either $b_{3}$ is the rightmost vertex in $\sigma$ that sees $b_{2}$ and misses $x$ or $b_{3}$ is the rightmost vertex in $\sigma$ that sees $b_{2}$ and misses every $a_{r}, 1 \leq r \leq i$.

Then we have the following:

1. If $i+1=3, x<a_{2}<b_{1}$ in $\sigma$, and $a_{i+1}$ is the rightmost vertex after $b_{1}$ in $\sigma$ such that $a_{i+1}$ sees $a_{i}$ but misses $x$, then $B\left(x, a_{i+1}, b_{j}\right)$ is a larger extended bull in which $x$ is the nose.

2. If $b_{j-1}<a_{i}<b_{j}$ in $\sigma$ and $a_{i+1}$ is the rightmost vertex after $b_{j}$ in $\sigma$ such that $a_{i+1}$ sees $a_{i}$ but misses $b_{j-1}$, then $B\left(x, a_{i+1}, b_{j}\right)$ is a larger extended bull in which $x$ is the nose.

3. If $j+1=3, x<b_{2}<a_{1}$ in $\sigma$, and $b_{j+1}$ is the rightmost vertex after $a_{1}$ in $\sigma$ such that $b_{j+1}$ sees $b_{j}$ but misses $x$, then $B\left(x, a_{i}, b_{j+1}\right)$ is a larger extended bull in which $x$ is the nose.

4. If $a_{i-1}<b_{j}<a_{i}$ in $\sigma$ and $b_{j+1}$ is the rightmost vertex after $a_{i}$ in $\sigma$ such that $b_{j+1}$ sees $b_{j}$ but misses $a_{i-1}$, then $B\left(x, a_{i}, b_{j+1}\right)$ is a larger extended bull in which $x$ is the nose.

Proof of Lemma 12, Let $G$ be a (building, gem)-free graph and $\sigma$ be an ordering of vertices of $G$ produced by LBFS. Suppose vertex $x$ is the nose of an extended bull in $H_{x}$ that satisfies the hypotheses of the lemma. We will prove Statements 1 and 2 simultaneously; the proofs of Statements 3 and 4 are similar. In the case of Statement 1, suppose $x<a_{2}<b_{1}$ in $\sigma$ and $a_{3}$ is the rightmost vertex after $b_{1}$ in $\sigma$ such that $a_{3}$ sees $a_{2}$ but misses $x$. In the case of Statement 2, suppose $b_{j-1}<a_{i}<b_{j}$ in $\sigma$ and $a_{i+1}$ is the 
rightmost vertex after $b_{j}$ in $\sigma$ such that $a_{i+1}$ sees $a_{i}$ but misses $b_{j-1}$. We will prove that $B\left(x, a_{i+1}, b_{j}\right)$ is a larger extended bull in which $x$ is the nose.

We need to establish that the new vertex $a_{i+1}$ misses $x$. This follows from the hypothesis in the case of Statement 1. For Statement 2 we have the following argument. Suppose $a_{i+1}$ sees $x$ and $b_{1}$. From the hypotheses we have the following: If $i+1=3$, then $a_{1}<a_{i+1}$; otherwise $a_{i}<a_{i+1}$. Now, if $a_{i+1}$ misses $b_{2}$, then $B\left(x, a_{2}, b_{2}\right)$ is not the rightmost bull in which $x$ is the nose; $\left\{x, a_{i+1}, a_{i}, b_{1}, b_{2}\right\}$ induces a bull that has a vertex to the right of a vertex of $B\left(x, a_{2}, b_{2}\right)$. So $a_{i+1}$ sees $b_{2}$. Now, if $a_{i+1}$ misses $a_{1}$, $\left\{b_{1}, b_{2}, a_{i+1}, x, a_{1}\right\}$ induces a gem in $G$; hence, $a_{i+1}$ must see $a_{1}$. If $i+1=3$, then $\left\{a_{3}, a_{2}, a_{1}, b_{1}, b_{2}\right\}$ induces a gem in $G$. So assume that $i+1 \geq 4$. Then $\left\{x, a_{1}, \ldots, a_{i+1}\right\}$ induces a near building in $G$, which by Claim 1 is a contradiction. So now suppose $a_{i+1}$ sees $x$ and misses $b_{1}$. If $a_{i+1}$ misses $a_{1}$, consider $r, 2 \leq r \leq i$, the smallest index such that $a_{i+1}$ sees $a_{r}$. Then $\left\{x, b_{1}, a_{i+1}, a_{1}, \ldots, a_{r}\right\}$ induces a building in $G$. So $a_{i+1}$ sees $a_{1}$. If $i+1=3$, $\left\{a_{1}, a_{2}, a_{3}, x, b_{1}\right\}$ induces a gem in $G$. If $i+1 \geq 4$, then $\left\{x, a_{1}, \ldots, a_{i+1}\right\}$ induces a near building in $G$. So we may conclude that $a_{i+1}$ misses $x$.

The remainder of the argument applies to Statements 1 and 2. Suppose $a_{i+1}$ sees $a_{1}$. Further suppose $a_{i+1}$ misses $b_{1}$. If $a_{i+1}$ sees $b_{2}, G$ has an induced building. If $a_{i+1}$ misses $b_{2}$, then $B\left(x, a_{2}, b_{2}\right)$ is not the rightmost bull in which $x$ is the nose; $\left\{x, a_{1}, a_{i+1}, b_{1}, b_{2}\right\}$ induces a bull that has a vertex to the right of a vertex of $B\left(x, a_{2}, b_{2}\right)$. Therefore, $a_{i+1}$ must see $b_{1}$. If $i+1=3$, then $\left\{a_{1}, a_{2}, a_{3}, b_{1}, x\right\}$ induces a gem in $G$. So assume that $i+1 \geq 4$. Then $\left\{b_{1}, a_{1}, \ldots, a_{i+1}\right\}$ induces a near building in $G$. So we may conclude that $a_{i+1}$ misses $a_{1}$.

Now we claim that $a_{i+1}$ misses all of $\left\{a_{1}, \ldots, a_{i-1}\right\}$. This is clearly the case if $i+1=3$, so assume that $i+1 \geq 4$. We have already established that $a_{i+1}$ misses $x$ and $a_{1}$. Suppose $a_{i+1}$ sees some vertex in $\left\{a_{2}, \ldots, a_{i-1}\right\}$. Let $r, 2 \leq r \leq i-1$, be the smallest index such that $a_{i+1}$ sees $a_{r}$. Suppose $r=2$ and $a_{3}$ is the rightmost vertex that sees $a_{2}$ and misses $x$. In this case, we have $a_{i+1}$ is a vertex to the right of $a_{3}$ that sees $a_{2}$ and misses $x$, which is a contradiction. Otherwise, $r \geq 3$ or $a_{3}$ is the rightmost vertex that sees $a_{2}$ and misses every $b_{r}, 1 \leq r \leq j$. In these cases, by the choice of $a_{r+1}$, $a_{i+1}$ sees some vertex in $\left\{b_{1}, \ldots, b_{j}\right\}$; otherwise, $a_{i+1}$ is a vertex to the right of $a_{r+1}$ that sees $a_{r}$ and misses all of $\left\{b_{1}, \ldots, b_{j}\right\}$. Let $s, 1 \leq s \leq j$, be the smallest index such that $a_{i+1}$ sees $b_{s}$. Now $\left\{x, a_{1}, \ldots, a_{r}, a_{i+1}, b_{1}, \ldots, b_{s}\right\}$ induces a building in $G$, which is a contradiction.

Finally, $a_{i+1}$ misses all of $\left\{b_{1}, \ldots, b_{j}\right\}$. Suppose not. Let $s, 1 \leq s \leq j$, be the smallest index such that $a_{i+1}$ sees $b_{s}$. Now $\left\{x, a_{1}, \ldots, a_{i+1}, b_{1}, \ldots, b_{s}\right\}$ induces a building in $G$, which is a contradiction. 
Hence, $B\left(x, a_{i+1}, b_{j}\right)$ is a larger extended bull in which $x$ is the nose.

Recall that if $\sigma$ is an LBFS ordering of graph $G$, then $H_{x}$ refers to the subgraph of $G$ induced by $x$ and the vertices to the right of $x$ in $\sigma$.

Theorem 3 Suppose $G$ is a (building, gem)-free graph and $\sigma$ is an LBFS ordering of $G$. Then, each vertex $v$ is not the nose of any bull in $H_{v}$.

Proof of Theorem 3. Let us suppose to the contrary that some vertex $x$ is the nose of a bull in $H_{x}$. Let $B\left(x, a_{2}, b_{2}\right)$ be the rightmost bull in $H_{x}$ in which $x$ is the nose. We will show that starting from $B\left(x, a_{2}, b_{2}\right)$, we can inductively grow larger and larger extended bulls in $H_{x}$ in which $x$ is the nose, contradicting the graph $G$ being finite. From here on, all the adjacencies referred to are with respect to the graph $H_{x}$.

Without loss of generality, we can assume $x<a_{1}<b_{1}$. First, we consider the twelve possible arrangements of the vertices $x, a_{1}, a_{2}, b_{1}$, and $b_{2}$ in $\sigma$ and for each arrangement we show that $H_{x}$ contains a specific larger extended bull $B\left(x, a_{i}, b_{j}\right)$. We do this so that every extended bull $B\left(x, a_{i}, b_{j}\right)$ that we develop is then conducive to the same inductive argument. Having done this basis step, we will use the inductive argument to show that any extended bull $B\left(x, a_{i}, b_{j}\right)$ can further be extended into either $B\left(x, a_{i+1}, b_{j}\right)$ or $B\left(x, a_{i}, b_{j+1}\right)$.

We repeatedly use $\left(P_{*}\right)$ on an extendable triple $(a, b, c)$ that is part of some extended bull $L$ to bring in a new vertex $d$ into the argument; for the sake of brevity, we say "use the triple $(a, b, c)$ " to refer to this process. When we do this, we will invoke Lemma 12 to conclude that the new vertex $d$ and the vertices of $L$ induce a larger extended bull. Also, after each extension, we designate a specific extendable triple $(x, y, z)$ as the "next triple". The idea is that in the next step of extension, the triple $(x, y, z)$ is to be used. We carefully choose such a next triple so as to ensure that the conditions of Lemma 12 are met. Finally, every time we bring in a new vertex $d$ while extending a triple $(a, b, c)$, we always choose the rightmost such $d$ in $\sigma$ that satisfies the conditions of selection. We now consider each of the twelve possibilities.

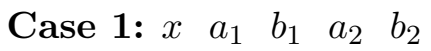

Use the triple $\left(b_{1}, a_{2}, b_{2}\right)$ and choose $a_{3}>b_{2}$ that sees $a_{2}$ and misses $b_{1}$. Apply Lemma 12 to get the larger extended bull $B\left(x, a_{3}, b_{2}\right)$ and the sequence

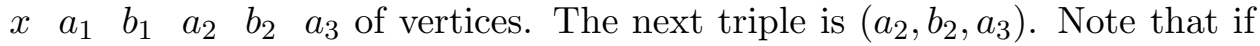
this triple is used to choose $b_{3}>a_{3}$ that sees $b_{2}$ and misses $a_{2}$, then $b_{1}<b_{3}$ will hold.

Case 2: $x \quad a_{1} \quad b_{1} \quad b_{2} \quad a_{2}$

Use the triple $\left(a_{1}, b_{2}, a_{2}\right)$ and choose $b_{3}>a_{2}$ that sees $b_{2}$ and misses $a_{1}$. 
Apply Lemma 12 to get the extended bull $B\left(x, a_{2}, b_{3}\right)$ and the sequence

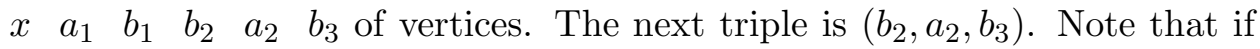
this triple is used to choose $a_{3}>b_{3}$ that sees $a_{2}$ and misses $b_{2}$, then $a_{1}<a_{3}$ will hold.

Case 3: $x$ alllllll $a_{1} b_{2} b_{2}$

Use the triple $\left(x, a_{2}, b_{1}\right)$ to choose $a_{3}>b_{1}$ that sees $a_{2}$ and misses $x$. By Lemma 12, $B\left(x, a_{3}, b_{2}\right)$ is a larger extended bull. We divide into two subcases based on the resulting sequence of vertices.

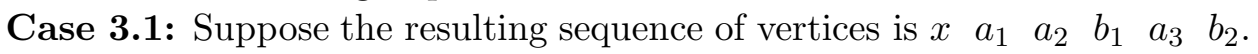
Use the triple $\left(b_{1}, a_{3}, b_{2}\right)$ and choose $a_{4}>b_{2}$ that sees $a_{3}$ and misses $b_{1}$. Apply Lemma 12 to get the extended bull $B\left(x, a_{4}, b_{2}\right)$. The resulting sequence is $x \quad a_{1} \quad a_{2} \quad b_{1} \quad a_{3} \quad b_{2} \quad a_{4}$ and the next triple is $\left(a_{3}, b_{2}, a_{4}\right)$. Note that if this triple is used to choose $b_{3}>a_{4}$ that sees $b_{2}$ and misses $a_{3}$, then $b_{1}<b_{3}$ will hold.

Case 3.2: Suppose the sequence of vertices is $x \quad a_{1} \quad a_{2} \quad b_{1} b_{2} a_{3}$. Then, the next triple is $\left(a_{2}, b_{2}, a_{3}\right)$. Note that if this triple is used to choose $b_{3}>a_{3}$ that sees $b_{2}$ and misses $a_{2}$, then $b_{1}<b_{3}$ will hold.

Case 4: $x$ a $a_{1} \quad b_{2} \quad b_{1} a_{2}$

Use the triple $\left(a_{1}, b_{2}, a_{2}\right)$ and choose $b_{3}>a_{2}$ that sees $b_{2}$ and misses $a_{1}$. Apply Lemma 12 to get the extended bull $B\left(x, a_{2}, b_{3}\right)$ and the sequence

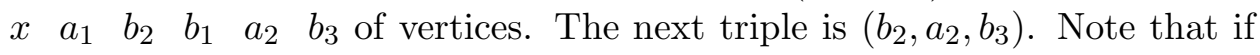
this triple is used to choose $a_{3}>b_{3}$ that sees $a_{2}$ and misses $b_{2}$, then $a_{1}<a_{3}$ will hold.

Case 5: $x \quad a_{1} \quad a_{2} \quad b_{2} \quad b_{1}$

Use the triple $\left(x, a_{2}, b_{1}\right)$ and choose $a_{3}>b_{1}$ that sees $a_{2}$ and misses $x$. Apply Lemma 12 to get the extended bull $B\left(x, a_{3}, b_{2}\right)$ and the sequence

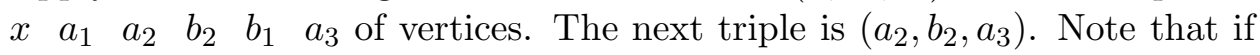
this triple is used to choose $b_{3}>a_{3}$ that sees $b_{2}$ and misses $a_{2}$, then $b_{1}<b_{3}$ will hold.

Case 6: $x \quad a_{1} \quad b_{2} \quad a_{2} \quad b_{1}$

Use the triple $\left(a_{1}, b_{2}, a_{2}\right)$ and choose $b_{3}>a_{2}$ that sees $b_{2}$ and misses $a_{1}$. Apply Lemma 12 to get the extended bull $B\left(x, a_{2}, b_{3}\right)$. We subdivide this case based on the resulting sequence of vertices.

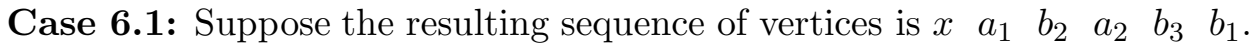
Use the triple $\left(x, a_{2}, b_{1}\right)$ and choose $a_{3}>b_{1}$ that sees $a_{2}$ and misses $x$. Apply Lemma 12 to get the extended bull $B\left(x, a_{3}, b_{3}\right)$. The resulting sequence is $\begin{array}{lllllllll}x & a_{1} & b_{2} & a_{2} & b_{3} & b_{1} & a_{3}\end{array}$ and the next triple is $\left(a_{2}, b_{3}, a_{3}\right)$.

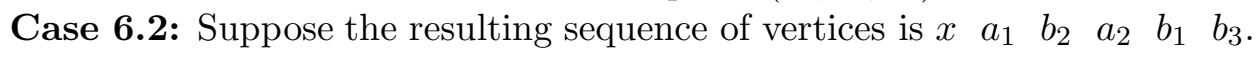
Use the triple $\left(x, a_{2}, b_{1}\right)$ and choose $a_{3}>b_{1}$ that sees $a_{2}$ and misses $x$. Apply Lemma 12 to get the extended bull $B\left(x, a_{3}, b_{3}\right)$. If the resulting sequence is 


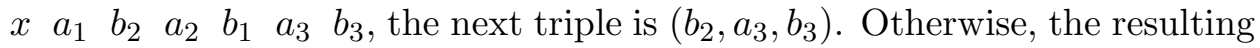
sequence is $x \quad a_{1} \quad b_{2} \quad a_{2} \quad b_{1} \quad b_{3} \quad a_{3}$ and the next triple is $\left(a_{2}, b_{3}, a_{3}\right)$.

Case 7: $x$ a $a_{2} \quad a_{1} \quad b_{1} b_{2}$

Use the triple $\left(x, a_{2}, b_{1}\right)$ to choose $a_{3}>b_{1}$ that sees $a_{2}$ and misses $x$. Apply Lemma 12 to get the extended bull $B\left(x, a_{3}, b_{2}\right)$. We subdivide based on the resulting sequence.

Case 7.1: Suppose the resulting sequence is $x a_{2} \quad a_{1} \quad b_{1} a_{3} \quad b_{2}$. Use the triple $\left(b_{1}, a_{3}, b_{2}\right)$ and choose $a_{4}>b_{2}$ that sees $a_{3}$ and misses $b_{1}$. Apply Lemma 12 to get the extended bull $B\left(x, a_{4}, b_{2}\right)$ and the sequence $x \quad a_{2} a_{1} b_{1} a_{3} b_{2} a_{4}$. The next triple is $\left(a_{3}, b_{2}, a_{4}\right)$. Note that if this triple is used to choose $b_{3}>a_{4}$ that sees $b_{2}$ and misses $a_{3}$, then $b_{1}<b_{3}$ will hold.

Case 7.2: Suppose the resulting sequence is $x \quad a_{2} \quad a_{1} b_{1} b_{2} a_{3}$. Then, the next triple is $\left(a_{2}, b_{2}, a_{3}\right)$. Note that if this triple is used to choose $b_{3}>a_{3}$ that sees $b_{2}$ and misses $a_{2}$, then $b_{1}<b_{3}$ will hold.

Case 8: $x \quad a_{2} \quad a_{1} \quad b_{2} \quad b_{1}$

Use the triple $\left(x, a_{2}, b_{1}\right)$ and choose $a_{3}>b_{1}$ that sees $a_{2}$ and misses $x$. Apply Lemma 12 to get the larger extended bull $B\left(x, a_{3}, b_{2}\right)$. The resulting sequence is $x \quad a_{2} \quad a_{1} \quad b_{2} \quad b_{1} \quad a_{3}$ and the next triple is $\left(a_{2}, b_{2}, a_{3}\right)$. Note that if this triple is used to choose $b_{3}>a_{3}$ that sees $b_{2}$ and misses $a_{2}$, then $b_{1}<b_{3}$ will hold.

Case 9: $x$ blllllll Use the triple $\left(x, b_{2}, a_{1}\right)$ and choose $b_{3}>a_{1}$ that sees $b_{2}$ and misses $x$. Apply Lemma 12 to get the larger extended bull $B\left(x, a_{2}, b_{3}\right)$. There are three possibilities for the resulting sequence.

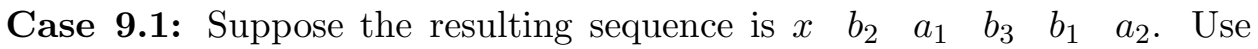
the triple $\left(a_{1}, b_{3}, a_{2}\right)$ and choose $b_{4}>a_{2}$ that sees $b_{3}$ that misses $a_{1}$. Apply Lemma 12 to get the extended bull $B\left(x, a_{2}, b_{4}\right)$ and the sequence $x \quad b_{2} a_{1} b_{3} b_{1} a_{2} b_{4}$. The next triple is $\left(b_{3}, a_{2}, b_{4}\right)$. Note that if this triple is used to choose $a_{3}>b_{4}$ that sees $a_{2}$ and misses $b_{3}$, then $a_{1}<a_{3}$ will hold.

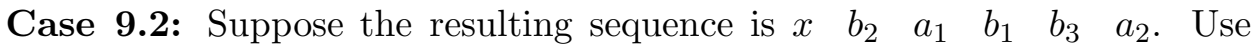
the triple $\left(a_{1}, b_{3}, a_{2}\right)$ and choose $b_{4}>a_{2}$ that sees $b_{3}$ that misses $a_{1}$. Apply Lemma 12 to get the extended bull $B\left(x, a_{2}, b_{4}\right)$ and the sequence $x \quad b_{2} a_{1} b_{1} b_{3} a_{2} b_{4}$. The next triple is $\left(b_{3}, a_{2}, b_{4}\right)$. Note that if this triple is used to choose $a_{3}>b_{4}$ that sees $a_{2}$ and misses $b_{3}$, then $a_{1}<a_{3}$ will hold.

Case 9.3: Suppose the resulting sequence is $x \quad b_{2} \quad a_{1} \quad b_{1} a_{2} \quad b_{3}$. The next triple is $\left(b_{2}, a_{2}, b_{3}\right)$. Note that if this triple is used to choose $a_{3}>b_{3}$ that sees $a_{2}$ and misses $b_{2}$, then $a_{1}<a_{3}$ will hold.

Case 10: $x$ blllll $\quad b_{2} \quad a_{1} \quad b_{1}$

Use the triple $\left(x, b_{2}, a_{1}\right)$ to choose $b_{3}>a_{1}$ that sees $b_{2}$ and misses $x$. Use Lemma 12 to get the larger extended bull $B\left(x, a_{2}, b_{3}\right)$. Again, there are 
three possible resulting sequences.

Case 10.1: Suppose the resulting sequence is $x \quad b_{2} a_{1} \quad b_{3} a_{2} b_{1}$. Use the triple is $\left(a_{1}, b_{3}, a_{2}\right)$ and choose $b_{4}>a_{2}$ that sees $b_{3}$ and misses $a_{1}$. Apply Lemma 12 to get the extended bull $B\left(x, a_{2}, b_{4}\right)$. The resulting sequence is

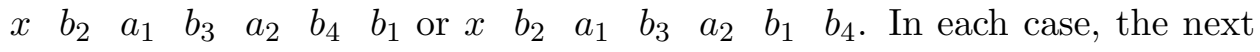
triple is $\left(b_{3}, a_{2}, b_{4}\right)$. Note that if this triple is used to choose $a_{3}>b_{4}$ that sees $a_{2}$ and misses $b_{3}$, then $a_{1}<a_{3}$ will hold.

Case 10.2: Suppose the resulting sequence is $x \quad b_{2} \quad a_{1} a_{2} b_{3} b_{1}$. The next triple is $\left(b_{2}, a_{2}, b_{3}\right)$.

Case 10.3: Suppose the resulting sequence is $x \quad b_{2} \quad a_{1} a_{2} b_{1} \quad b_{3}$. The next triple is $\left(b_{2}, a_{2}, b_{3}\right)$.

Note that in the last two subcases, if the next triple is used to choose $a_{3}>b_{3}$ that sees $a_{2}$ and misses $b_{2}$, then $a_{1}<a_{3}$ will hold.

Case 11: $x$ a $a_{2} \quad b_{2} \quad a_{1} b_{1}$

Use the triple $\left(x, a_{2}, b_{1}\right)$ to choose $a_{3}>b_{1}$ that sees $a_{2}$ and misses $x$. Use Lemma 12 to get the extended bull $B\left(x, a_{3}, b_{2}\right)$. The resulting sequence is $\begin{array}{lllllllll}x & a_{2} & b_{2} & a_{1} & b_{1} & a_{3}\end{array}$ and the next triple is $\left(a_{2}, b_{2}, a_{3}\right)$. Note that if this triple is used to choose $b_{3}>a_{3}$ that sees $b_{2}$ and misses $a_{2}$, then $b_{1}<b_{3}$ will hold.

Case 12: $x$ blllll $b_{2} \quad a_{2} \quad a_{1} b_{1}$

Use the triple $\left(x, b_{2}, a_{1}\right)$ and choose $b_{3}>a_{1}$ that sees $b_{2}$ and misses $x$. Apply Lemma 12 to get the extended bull $B\left(x, a_{2}, b_{3}\right)$. The resulting sequence is either $x \quad b_{2} \quad a_{2} \quad a_{1} \quad b_{3} \quad b_{1}$ or $x \quad b_{2} \quad a_{2} \quad a_{1} b_{1} \quad b_{3}$. In each case, the next triple is $\left(b_{2}, a_{2}, b_{3}\right)$. Again, if this triple is used to choose $a_{3}>b_{3}$ that sees $a_{2}$ and misses $b_{2}$, then $a_{1}<a_{3}$ will hold.

Note that any next triple is either of the form $\left(a_{i-1}, b_{j}, a_{i}\right)$ or $\left(b_{j-1}, a_{i}, b_{j}\right)$; without loss of generality, assume that it is the latter and we already have the extended bull $B\left(x, a_{i}, b_{j}\right)$. Apply $\left(P_{*}\right)$ to the triple $\left(b_{j-1}, a_{i}, b_{j}\right)$ to choose the rightmost vertex $a_{i+1}$ such that $a_{i+1}>b_{j}$ and $a_{i+1}$ sees $a_{i}$ but misses $b_{j-1}$.

Observe that either $a_{3}$ is brought in as the rightmost vertex that sees $a_{2}$ and misses $x$ or it is the rightmost vertex that sees $a_{2}$ and misses some $b_{r}$. In the former case, $a_{3}$ satisfies the conditions of the Lemma 12, Otherwise, consider the general case. Assume $a_{p}, 3 \leq p \leq i$, is brought in as the rightmost vertex that sees $a_{p-1}$ and misses some $b_{q}$. Given that $B\left(x, a_{i}, b_{j}\right)$ is an extended bull, $a_{p}$ clearly misses every $b_{q}$. Therefore, it can be deduced that $a_{p}$ is indeed the rightmost vertex that sees $a_{p-1}$ and misses every $b_{q}$. Thus, every $a_{p}$ satisfies conditions of Lemma 12, By symmetry, every $b_{q}$ also satisfies the conditions of Lemma 12 .

Applying Lemma 12, we get the larger extended bull $B\left(x, a_{i+1}, b_{j}\right)$. As we now have $a_{i}<b_{j}<a_{i+1}$, we can use $\left(a_{i}, b_{j}, a_{i+1}\right)$ as the next triple to 
complete the inductive argument.

Corollary 2 Suppose $G$ is a (building, gem)-free graph. Then, every induced subgraph $H$ of $G$ contains a vertex $v$ such that $v$ is not the nose of any bull in $H$.

Figure 2 shows that without forbidding the gem, Theorem 3 cannot be proved. For a vertex $v$ in graph $G$, let $D_{2}(v)$ denote the set of those vertices of $G$ that are within distance two from $v$. The following theorem was proved in [6].

Theorem 4 [6] Graph $G$ is distance hereditary if and only if for every LBFS ordering $v_{1} v_{2} \ldots v_{n}$ of $G$ and for each $v_{i}, D_{2}\left(v_{i}\right)$ in $G\left[v_{i}, \ldots, v_{n}\right]$ does not contain a $P_{4}$.

If $v$ is a vertex of $G$ such that $D_{2}(v)$ does not contain a $P_{4}$, then $v$ is not the nose of any bull in $G$. However, the converse is not true; consider the graph constructed by starting with the $P_{4}$ abcd and adding vertex $v$ adjacent only to $a$ and $c$. Therefore, the elimination scheme addressed in Theorem 3 is more general than the one given in Theorem 4 .

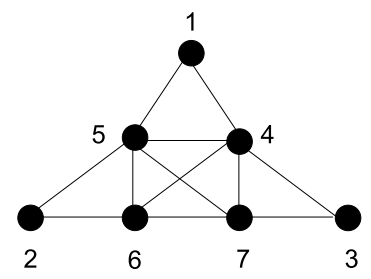

Figure 2: A building-free graph with an LBFS ordering where the first vertex is the nose of a bull.

In view of Corollary 2, let $\mathcal{C}$ denote the class of building-free graphs $G$ such that in every induced subgraph $H$ of $G$ there exists a vertex that is not nose of any bull in $H$. What can we say about the class $\mathcal{C}$ ? Every strongly chordal graph belongs to the class $\mathcal{C}$. More generally, every strongly 
orderable graph [5] that does not contain a building belongs to the class $\mathcal{C}$. Clearly, any $k$-sun, $k \geq 4$, is a minimal forbidden subgraph for the class $\mathcal{C}$. Conversely, what are minimal graphs in which every vertex is nose of some bull? Figure 3 is $\left(C_{9}\right)^{2}$, which is minimal for the property. We note that this graph contains buildings.

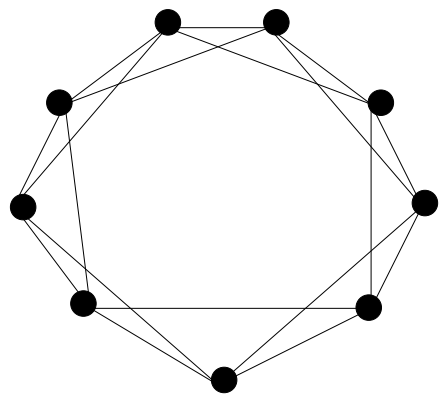

Figure 3: In $\left(C_{9}\right)^{2}$ every vertex is nose of a bull.

\section{Discussion}

We conclude the paper with some problems. A module $M$ in graph $G=$ $(V, E)$ is a set of vertices such that $M \neq \emptyset, M \neq V$, and for every vertex $x \in V-M$, either $x$ sees every vertex in $M$ or $x$ misses every vertex in $M$. The use of modules in the design of efficient algorithms is a well-researched subject [2]. It was proved in [11] that every hhd-free graph contains a module or a simplicial vertex; this result has been used to design an efficient recognition algorithm for the class of hhd-free graphs [12. In view of this, is it true that every hhds-free graph contains a module or a simple vertex?

Given that a sun in a graph can be found efficiently when the graph belongs to some perfect graph classes, what is the complexity of finding a sun in a weakly chordal graph? A graph is weakly chordal if neither the graph nor its complement contains any holes. More generally, what is the complexity of finding a sun in a perfect graph? 


\section{References}

[1] Alon, N., Yuster, R., Zwick, U.: Finding and counting given length cycles. Algorithmica 17, 209-223 (1997)

[2] Brandstädt, A., Le, V.B., Spinrad, J.P.: Graph Classes: A survey. SIAM Monographs on Discrete Mathematics and Applications (1999)

[3] Chvátal, V.: Perfectly ordered graphs. In: Topics on perfect graphs. Berge, C., Chvátal, V., (eds). North-Holland, Amsterdam, 63-65 (1984)

[4] Dirac, G.A: On rigid circuit graphs. Abh. Math. Sem. Univ. Hamburg 25, 71-76 (1961)

[5] Dragan, F.: Strongly orderable graphs: a common generalization of strongly chordal and chordal bipartite graphs. Discrete Applied Mathematics 99, 427-442 (2000)

[6] Dragan, F., Nicolai, F.: LexBFS-orderings of distance-hereditary graphs. Schriftenreihe des Fachbereichs Mathematik der Universität Duisburg, Duisburg, Germany, SM-DU-303 (1995)

[7] Eschen, E.M., Hoàng, C.T., Sritharan, R.: An $\mathrm{O}\left(n^{3}\right)$-time recognition algorithm for hhds-free graphs. Graphs and Combinatorics 23, 209-231 (2007)

[8] Farber, M.: Characterizations of strongly chordal graphs. Discrete Mathematics 43, 173-189 (1983)

[9] Golumbic, M.C.: Algorithmic graph theory and perfect graphs. Academic Press, (1980).

[10] Hoàng, C.T: On the complexity of finding a sun in a graph. To appear in SIAM Journal on Discrete Mathematics.

[11] Hoàng, C.T., Khouzam, N.: On brittle graphs. Journal of Graph Theory 12, 391-404 (1988)

[12] Hoàng, C.T., Sritharan, R.: Finding houses and holes in graphs. Theoretical Computer Science 259, 233-244 (2001)

[13] Nikolopoulos, S.D., Palios, L.: Recognizing hhds-free graphs. In: Proceedings of the $31^{\text {st }}$ International Workshop on Graph Theoretic Concepts in Computer Science (WG 2005), Metz, France, (2005) 
[14] Nikolopoulos, S.D., Palios, L.: Recognizing hh-free, hhd-free, and Welsh-Powell opposition graphs. Discrete Mathematics and Theoretical Computer Science 8, 65-82 (2006)

[15] Paige, R., Tarjan, R.E.: Three partition refinement algorithms. SIAM Journal on Computing 16, 973-989 (1987)

[16] Rose, D.J., Tarjan, R.E., Leuker, G.S.: Algorithmic aspects of vertex elimination on graphs. SIAM Journal on Computing 5, 266-283 (1976)

[17] Spinrad, J.P.: Doubly lexical ordering of dense 0/1 matrices, Information Processing Letters 45, 229-235 (1993) 